\title{
Effect of donor parameters on primary graft failure and the recovery of acuity after keratoplasty
}

\author{
Brett L Halliday, Sybil A Ritten
}

\begin{abstract}
A retrospective evaluation was made of 983 penetrating corneal grafts. Donor corneas stored in either K-Sol or McCarey-Kaufman media had a significantly greater rate of primary graft failure (about $2 \%$ ) than those kept in moist chamber storage $(0 \%)$. Selected subgroups of $\mathbf{5 0}$ corneas from each storage system were studied to determine the time taken to reach a postoperative acuity of $6 / 12$. No significant correlation was found between this time and either the duration of storage of donor corneas or the age of donor. A significant delay in recovery of visual acuity was found with increasing age of recipient. The increased average time from donor death to surgery for K-Sol (56 hours) and McCareyKaufman (40 hours) corneas compared with moist chamber corneas ( 15 hours) facilitated the scheduling of surgery at the cost of increased chance of primary graft failure.
\end{abstract}

Factors which increase the likelihood of success in penetrating keratoplasty include good quality donor material, minimal surgical trauma, and a favourable host environment. There is no simple measure of donor tissue quality. Many surgeons are influenced in their choice of material by such attributes as the age and the time elapsed since donor death. It is commonly believed that the best results, in terms of early graft clarity, are obtained by using corneas from young donors with minimum delay after death. However, in one series of nearly 400 cases no significant difference in subsequent graft clarity was found between donors grouped into four age bands (from under 50 years to over 70 years old). ${ }^{1}$ Similar results were seen in the Australian Graft Registry; in nearly 500 cases the probability of graft survival was almost identical irrespective of donor age of less than 50 years or greater than 70 years. ${ }^{2}$ The Australian Graft Registry also reported that storage of corneas in McCareyKaufman medium for as long as five days had no effect on subsequent graft survival. The duration of corneal storage (of up to three days in McCarey-Kaufman medium and up to a few weeks in organ culture) was unrelated to the postoperative visual acuity in one small series. ${ }^{3}$ The use of storage media allows time for tissue typing for matched grafts, screening of donor serum for viral antibodies, and for optimum scheduling of surgery.

The use of storage media involves extra handling of the donor cornea and subjecting it to a change of environment. It is possible that this may result in an increased rate of primary graft failure compared with moist chamber storage. It is also possible, however, that storage media are truly preservative. Perhaps eyes in moist chamber storage should be transferred to a storage medium even if their use is envisaged within a few hours. Media that contain chondroitin sulphate, such as K-Sol, ${ }^{4}$ appear to be more effective at maintaining higher levels of adenosine triphosphate (ATP), necessary for the cornea to remain viable, than storage media without, such as McCarey-Kaufman, which in turn is more effective than moist chamber storage. ${ }^{56}$ In animal experiments ${ }^{7}$ and in vitro with human corneas ${ }^{8} \mathrm{~K}$-Sol appears to be superior to McCarey-Kaufman medium in maintaining endothelial cell viability.

This study was designed to compare the rate of primary graft failure of corneas stored in K-Sol or McCarey-Kaufman media with those stored in a moist chamber. It also was designed to examine the possible relationship between the known donor cornea parameters, such as age of donor and method and time of storage, and the postoperative clinical performance of the cornea as represented by the time taken for recovery of visual acuity to $6 / 12$.

\section{Materials and methods}

A retrospective study was made of all corneal grafts performed at Moorfields Eye Hospital from January 1984 to October 1988. Until August 1985 the only method of storage used was the whole eye, moist chamber technique. At this time McCarey-Kaufman medium was introduced, and then in April 1987 a change was made to K-Sol. Donor corneas were all examined with a slit-lamp and most had endothelial specular microscopy before they were passed as being suitable for storage in McCarey Kaufman or K-Sol Media.

The incidence of primary graft failure was determined for all 983 patients. This was diagnosed when, in the absence of an identifiable cause such as glaucoma or rejection, a graft remained oedematous and failed to show any postoperative recovery of function.

For the assessment of the recovery of visual acuity strict exclusion criteria were employed. These were designed to minimise, as far as possible, the influence of factors other than corneal clarity on the recovery of visual acuity. Eyes were excluded from the study if there was known to be any noteworthy preoperative path-

TABLE I Numbers of primary graft failures

\begin{tabular}{|c|c|c|c|}
\hline \multirow[b]{2}{*}{ Graft patients } & \multicolumn{3}{|c|}{ Number $(\%)$ of patients } \\
\hline & $\begin{array}{l}\text { Moist } \\
\text { chamber }\end{array}$ & $\begin{array}{l}\text { McCarey- } \\
\text { Kaufman }\end{array}$ & $K$-Sol \\
\hline $\begin{array}{l}\text { Selected } 50 \text { patients } \\
\text { All graft patients }\end{array}$ & $\begin{array}{l}0 / 50(0) \\
0 / 278(0)\end{array}$ & $\begin{array}{l}1 / 50(2 \cdot 0) \\
4 / 224(1 \cdot 78)\end{array}$ & $\begin{array}{c}3 / 50(6 \cdot 0) \\
10 / 481(2 \cdot 07)\end{array}$ \\
\hline
\end{tabular}

City Road, London EC1V 2PD B L Halliday

Correspondence to: B L Halliday, FRCS 
TABLE II Summary of findings in primary failure grafts

\begin{tabular}{|c|c|c|c|c|c|c|}
\hline Case & Storage & $\begin{array}{l}\text { Recipient } \\
\text { age } \\
\text { (years) }\end{array}$ & $\begin{array}{l}\text { Recipient } \\
\text { diagnosis }\end{array}$ & $\begin{array}{l}\text { Donor } \\
\text { age } \\
\text { (years) }\end{array}$ & $\begin{array}{l}\text { Time from } \\
\text { death to } \\
\text { storage } \\
\text { (hours) }\end{array}$ & $\begin{array}{l}\text { Time in } \\
\text { storage } \\
\text { (hours) }\end{array}$ \\
\hline 1 & McCarey-Kaufmam & 23 & Keratoconus & 77 & 21 & 25 \\
\hline 2 & McCarey-Kaufman & 64 & Lattice dystrophy & 73 & 9 & 48 \\
\hline 3 & McCarey-Kaufman & 51 & Pseudophakia & 67 & 12 & 5 \\
\hline $4^{\star}$ & McCarey-Kaufman & 30 & Measles keratitis & 61 & 13 & 21 \\
\hline $5^{\star}$ & K-Sol & 77 & Interstitial keratitis & 65 & 13 & 110 \\
\hline $6^{\star}$ & K-Sol & 25 & Macular dystrophy & 69 & 19 & 67 \\
\hline 7 & K-Sol & 72 & Pseudophakia & 69 & 19 & 72 \\
\hline 8 & K-Sol & 68 & Traumatic scar & 70 & 23 & 90 \\
\hline $9^{\star}$ & K-Sol & 62 & Fuchs dystrophy & 70 & 23 & 117 \\
\hline 10 & K-Sol & 60 & Pseudophakia & 40 & 21 & 48 \\
\hline 11 & K-Sol & 85 & Pseudophakia & 67 & 17 & 158 \\
\hline 12 & K-Sol & 63 & Fuchs dystrophy & 59 & 2 & 67 \\
\hline 13 & K-Sol & 72 & Fuchs dystrophy & 72 & 14 & 77 \\
\hline 14 & K-Sol & 40 & Keratoconus & 27 & 32 & 51 \\
\hline
\end{tabular}

^These patients were from the study groups of 50 patients.

ology in the recipient apart from corneal opacity. Eyes with corneal vascularisation, poor tear film, corneal perforation, or with pre-existing glaucoma or retinal pathology were excluded. Eyes that had intracapsular cataract surgery performed at graft surgery were excluded, as this procedure is known to be associated with a high incidence of cystoid macular oedema, ${ }^{9}$ but eyes that had extracapsular cataract surgery, with or without lens implant, at the time of corneal graft were included.

Eyes were also excluded from this part of the study if conditions likely to affect visual acuity were noted after graft surgery, such as retinal detachment, cystoid macular oedema, or glaucoma. This 'retrospective' exclusion of cases is justifiable because these complications cannot be related to the donor cornea parameters.

After these exclusions the first 50 grafts from each of the three storage methods were included in the recovery of vision study. For these 150 patients the time taken to reach a postoperative acuity of 6/12 (with refraction or with the use of a pinhole) was recorded. Other recorded details included donor and recipient ages, time to storage, and time from storage to surgery, the use of viscoelastic material ( $2 \%$ hydroxypropylmethylcellulose or sodium hyaluronate), and whether surgery was performed by a consultant or by a resident. Hospital notes were also examined to find patients who had visual acuities recorded, at successive clinic visits, with the use of a pinhole and with refraction. These visual acuities, and the interval between them, were recorded in order to compare these two techniques of optical correction.

\section{Results}

Table I shows the incidence of primary graft

TABLE III Indication for corneal graft

\begin{tabular}{|c|c|c|c|}
\hline \multirow[b]{2}{*}{ Diagnosis } & \multicolumn{3}{|c|}{ Number of patients ${ }^{\star}$} \\
\hline & $\begin{array}{l}\text { Moist } \\
\text { chamber }\end{array}$ & $\begin{array}{l}\text { McCarey- } \\
\text { Kaufman }\end{array}$ & K-Sol \\
\hline $\begin{array}{l}\text { Keratoconus } \\
\text { Age related corneal decompensation; Fuchs, pseudophakic, aphakic } \\
\text { Corneal dystrophies; lattice, macular, granular, congenital hereditary } \\
\text { Interstitial keratitis } \\
\text { Others } \\
\text { Total }\end{array}$ & $\begin{array}{l}33 \\
8(2) \\
2 \\
3(2) \\
4(2) \\
50\end{array}$ & $\begin{array}{l}27(1) \\
5(4) \\
6 \\
4(1) \\
8 \\
50\end{array}$ & $\begin{array}{l}24 \\
11(2) \\
7(3) \\
2(2) \\
6 \\
50\end{array}$ \\
\hline
\end{tabular}

^Figures in brackets indicate number in each group where cataract surgery was performed at the time of corneal graft. failures, both for the total number of grafts (278 moist chamber, 224 McCarey Kaufman and 481 $\mathrm{K}-\mathrm{Sol}$ ) and for the selected subgroups (each of 50 patients). There was no statistical difference between the three storage systems for the subgroups, but over the total number of grafts the rate of primary graft failure was significantly greater both for McCarey-Kaufman $(p<0.05)$ and for K-Sol $(\mathrm{p}<0.02)$ storage media compared with the moist chamber group ( $\chi^{2}$ test). Table II provides clinical details on the primary graft failures.

For the selected subgroups of 50 patients the average age of recipient was 40 years for the moist chamber group, 43 for McCarey-Kaufman group, and $\mathbf{4 5}$ for the $\mathrm{K}$-Sol group. The average time from death to storage was eight hours (range one to 22) for moist chamber, 10 hours (range two to 26) for McCarey-Kaufman, and 11 hours (range two to 23) for K-Sol. Surgery was performed by a consultant in $72 \%$ of the moist chamber group, $82 \%$ in the McCarey-Kaufman group, and $74 \%$ in the $\mathrm{K}$-Sol group. These differences are not significant. Viscoelastic material was used significantly more often in both the McCarey-Kaufman (64\%) and the $\mathrm{K}-\mathrm{Sol}(54 \%)$ groups than in the moist chamber group $(33 \%)\left(\chi^{2}\right.$ test, $\left.\mathrm{p}<0.02\right)$. The average time from donor death to surgery was also different between the three groups: 15 hours (range one to 34) for moist chamber storage, 40 hours (range 10 to 102) for McCarey-Kaufman, and 56 hours (range 18 to 188) for K-Sol. Table 3 compares the preoperative diagnoses in these three subgroups of patients.

In 200 randomly selected clinic visits a refraction was performed 18 times, representing one refraction per 11 visits. Fifty four occasions were found when a pinhole acuity recorded at one clinic visit was followed by refractive acuity at the subsequent visit (on average 1.24 months later). On average this pinhole acuity was 0.46 Snellen line worse than the subsequent refractive acuity. Sixteen occasions were found when a refractive acuity recorded at one clinic visit was followed by a pinhole acuity at the subsequent visit (on average 1.01 months later). This pinhole acuity was again, on average, worse than the refractive acuity, by 0.12 Snellen line. The refractions on these patients showed an average spherical error of 3.56 dioptres (range 0 to 12.75), with 4.99 dioptres of cylinder (range 0 to 10).

Figure 1 shows the recovery of visual acuity for each of the three selected subgroups. Each line plots the percentage of patients that, at a given time postoperatively, have achieved a visual acuity of 6/12. The plot is cumulative and does not show if a patient subsequently lost visual acuity. With the exception of the primary graft failures all eyes reached an acuity of $6 / 12$. There was no significant difference between the three groups (log rank test). The mean time to $6 / 12$ vision was $2 \cdot 3$ months for moist chamber, 2.6 months for McCarey-Kaufman, and 2.1 months for K-Sol. These differences are not significant.

Figures 2-4 show three scattergrams which plot the time to $6 / 12$ vision against the time from donor death to surgery for each of the three 


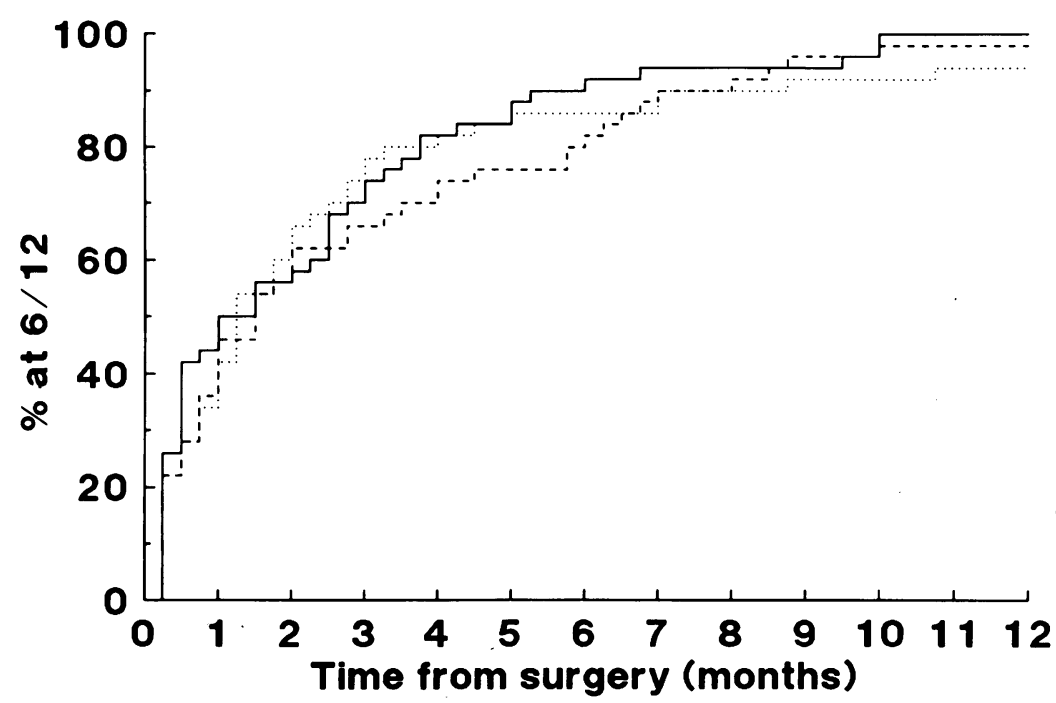

Figure 1: Cumulative percentage of patients that have achieved a visual acuity of 6/12 plotted against time. Solid line represents moist chamber storage, dashed line represents McCareyKaufman medium, and dotted line represents K-Sol.

storage systems. A linear regression line is shown on each scattergram, but in no case is the correlation significant; regression data are shown in Table 4. The negative slope for the moist chamber and McCarey-Kaufman plots indicates a (non-significant) reduced time to $6 / 12$ vision with longer storage times. For the K-Sol group the positive slope indicates a (non-significant) delay to $6 / 12$ vision of three days for every extra 24 hours that the cornea is kept in storage.

It is possible to make a direct comparison between the three storage techniques by considering donor eyes that were used between 18 and 34 hours after death. This time interval was common to all three storage techniques; 18 hours was the shortest period for K-Sol and 34 hours was the longest period for a moist chamber donor. Over this interval 18 moist chamber, 15 McCarey-Kaufman, and $16 \mathrm{~K}$-Sol stored corneas were used. There was no significant difference in average time to $6 / 12$ vision between these cases.

Figure 5 is a scattergram which plots the time to $6 / 12$ vision against age of the donor. Testing the three groups separately shows no significant correlation between time to $6 / 12$ vision and age of

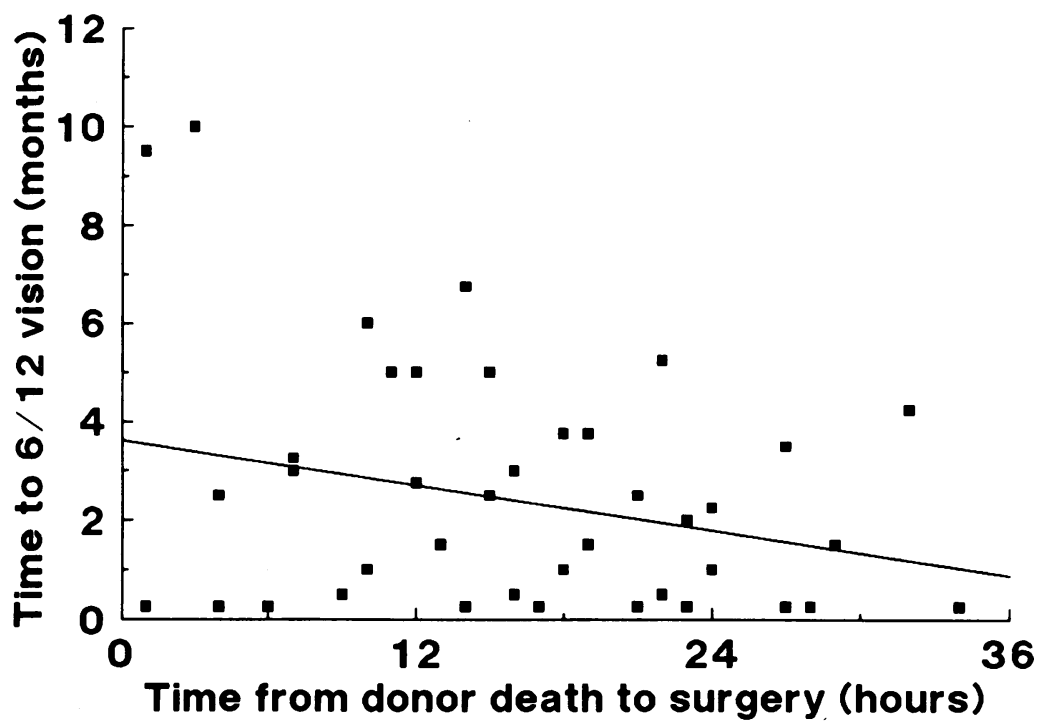

Figure 2: Moist chamber stored corneas. Scattergram of time to 6/12 vision versus time from donor death to surgery. The linear regression line shown is not significant. donor. Linear regression analysis on all three groups pooled shows no significant correlation (slope 0.0094 , intercept 1.94, correlation coefficient 0.061 ). This slope indicates a (nonsignificant) delay in time to $6 / 12$ vision of $0 \cdot 28$ days for each additional year of donor age. To examine the hypothesis that donors over the age of 60 may show a greater trend to poorer quality, linear regression analysis was repeated taking only those donors over the age of 60 . This again showed no significant correlation (slope -0.009 , intercept $3 \cdot 24$, correlation coefficient $-0 \cdot 026$ ). This slope indicates a (non-significant) reduction in time to $6 / 12$ vision with increasing donor age over 60 years.

Figure 6 is a scattergram which plots the time to $6 / 12$ vision against age of recipient. All three storage techniques are combined. Linear regression analysis showed a significant correlation ( $\mathrm{p}<0.025$, slope 0.027 , intercept $1 \cdot 17$, correlation coefficient of 0.22 ). The slope of the regression line represents a (significant) delay in time to $6 / 12$ vision of 0.83 days for each additional year of recipient age; a 20 -year old patient may be expected to reach $6 / 12$ vision six weeks earlier than a 70-year old. However, there was considerable overlap when confidence limits were considered; $95 \%$ of 20 -year old patients may be expected to reach 6/12 vision between two and 14 weeks, whereas $95 \%$ of 70 -year old patients took between three and 23 weeks.

\section{Discussion}

Over the total of 983 grafts there was a significant increase in the rate of primary graft failure for corneas stored in either McCarey-Kaufman or $\mathrm{K}$-Sol compared with those stored in a moist chamber. Over the selected 150 patients the rate of primary graft failure was greater in the media stored corneas, but this did not reach statistical significance. There was no difference in the rate of primary graft failure between the two media; each was associated with an incidence of about $2 \%$. In this series there were no primary graft failures in moist chamber stored corneas.

This superiority of moist chamber storage should be interpreted with caution. On average, McCarey-Kaufman and K-Sol corneas were stored respectively $2 \cdot 6$ and $3 \cdot 7$ times as long as moist chamber corneas; it may well be that even higher rates of failure would be associated with moist chamber storage over these lengths of time. It may be noted that viscoelastic material was used more often with the media stored corneas than with moist chamber corneas.

If the increased rate of primary graft failure with media storage reflects decreased endothelial cell viability, then it seems possible that such corneas may be slower to recover clarity, and so perhaps visual acuity, postoperatively. No such association was found; there was no significant difference between the three subgroups in either average time to $6 / 12$ vision or in the pattern of recovery, as shown in Figure 1. It may also be postulated that prolonged donor storage times and increased donor age should result in decreased endothelial cell viability and slower visual recovery. However, no significant correlation was found between the time to $6 / 12$ vision 


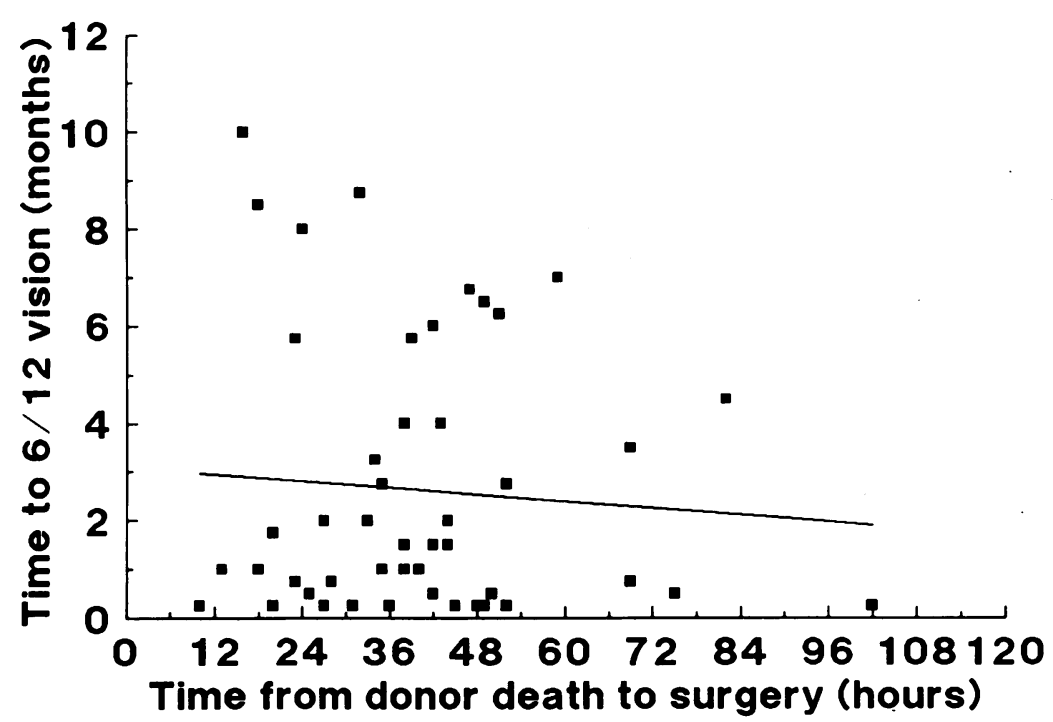

Figure 3: McCarey-Kaufman stored corneas. Scattergram of time to 6/12 vision versus time from donor death to surgery. The linear regression line shown is not significant.

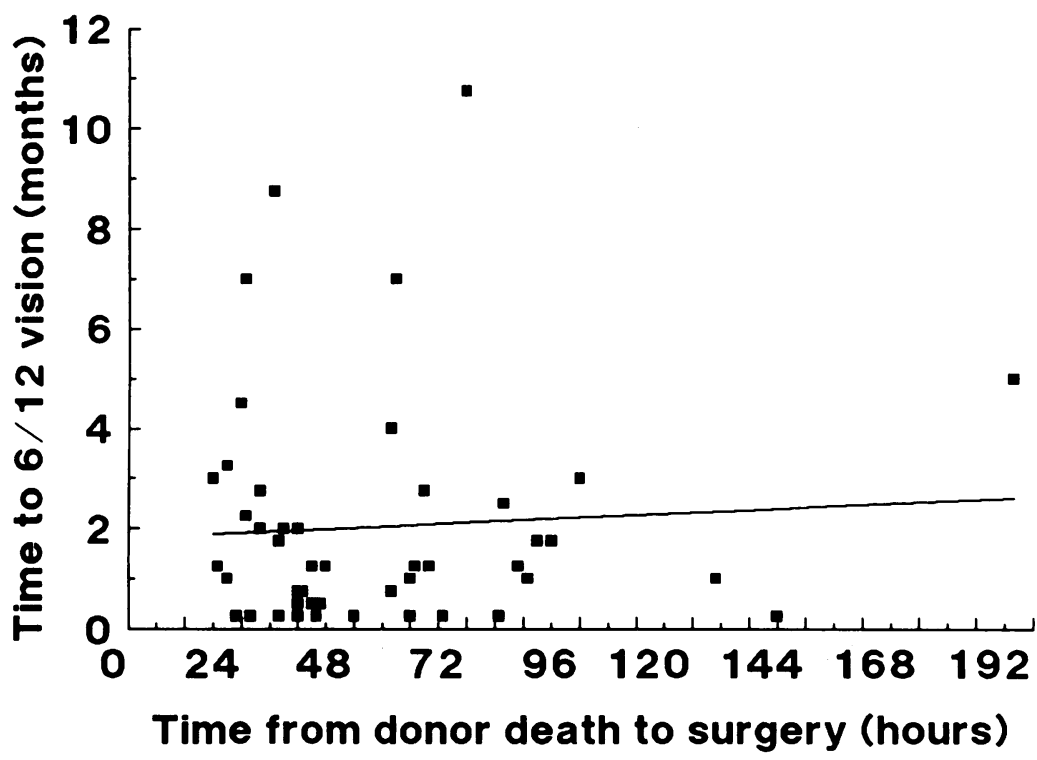

Figure 4: K-Sol stored corneas. Scattergram of time to 6/12 vision versus time from donor death to surgery. The linear regression line shown is not significant.

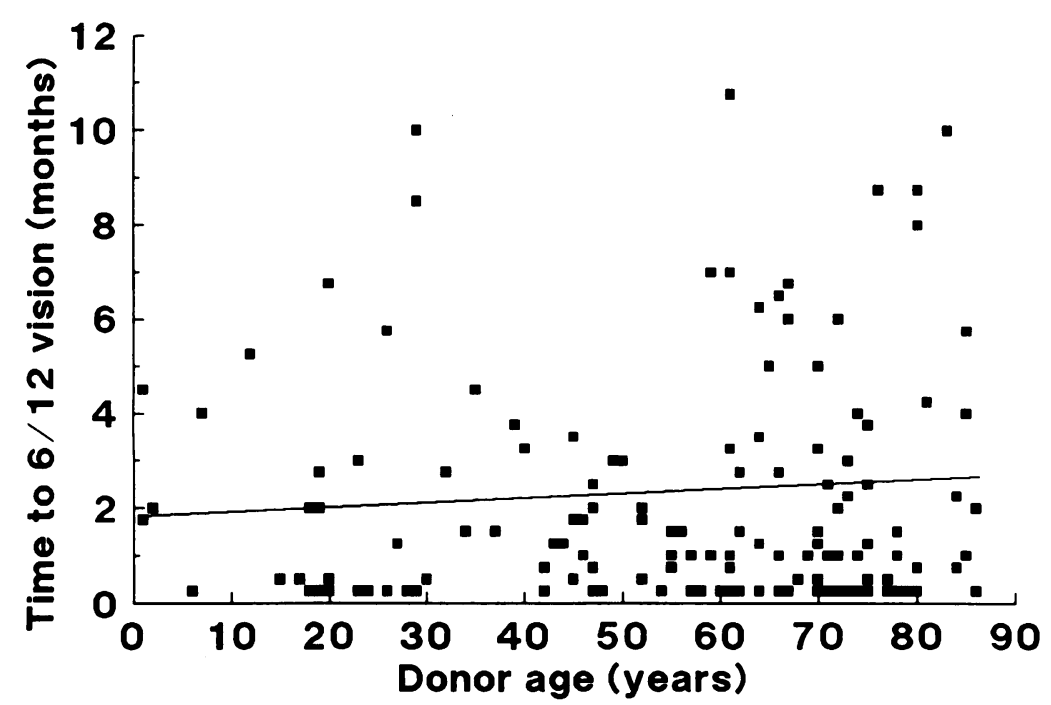

Figure 5: Scattergram of time to 6/12 vision versus age of donor. The linear regression line shown is not significant.
TABLE IV Regression data for Figure 2; months to 6/12 vision versus hours in storage

\begin{tabular}{lllll}
\hline & \multicolumn{3}{l}{ Regression data } \\
\cline { 2 - 5 } Storage type & Slope & Intercept & $\begin{array}{l}\text { Correlation } \\
\text { coefficient }\end{array}$ & Significance \\
\hline Moist chamber & -0.075 & 3.60 & -0.258 & NS \\
McCarey-Kaufman & -0.011 & 3.08 & -0.075 & NS \\
K-Sol & +0.004 & 1.81 & +0.063 & NS \\
\hline NS & & & &
\end{tabular}

$S=$ not significant

and either the time from death to surgery (Figs 2-4) or the donor age (Fig 5).

Prolonged storage does reduce endothelial cell density, ${ }^{10}$ and it is likely that endothelial cell viability is also affected by storage technique and donor age. The chosen clinical endpoint in this study, time to $6 / 12$ vision, is presumably not sensitive to such differences.

Although visual acuity is not a sine qua non of a functioning graft, it is usually true that an eye with good acuity has a successful, functioning graft. In this study visual acuity was used as a measure of graft quality. Patients who had additional pathology that could reduce acuity were excluded from the analysis. Good acuity does not, however, predict continuing graft success. Normal corneas have endothelial cell function in excess of that required to maintain corneal deturgescence and clarity. Techniques of storage and donor parameters that adversely affect endothelial cells may not reduce function below the critical value. An initially successful graft may, over a longer period of follow-up, lose clarity and fail.

Pinhole or refractive acuities were both considered satisfactory endpoints in this study. The large refractive errors often present in these patients resulted in poor unaided acuities which did not accurately reflect the corneal clarity. Unfortunately pinholes not only overcome refractive error," but they may also improve visual acuity in the presence of corneal irregularity and minor opacity. There therefore exists the possibility that the pinhole acuities recorded in this study presented an overoptimistic assessment of corneal clarity. The data showed, however, that the pinhole acuities were close to the refractive acuity (on average, within 0.38 Snellen lines). Pinhole acuities were actually slightly worse than refractive acuities, especially if the pinhole acuity was recorded at the visit before rather than the visit after the occasion when refraction was performed. The reduction in acuity measured with pinhole was more marked $(0.46$ Snellen lines) if the pinhole acuity was recorded before a refraction than when it was recorded after refraction ( $0 \cdot 12$ Snellen line). This difference may be explained by assuming that, with increasing postoperative time, corneal clarity improves and so allows the better acuity at the later date. For the pinhole acuity measured after refraction the small reduction in recorded acuity presumably represents the failure of the pinhole fully to correct the refractive error.

Donor cornea quality may also be assessed in vivo by measurements of corneal thickness and endothelial cell density. However these parameters do not provide an absolute standard by which the success of a graft may be judged. 


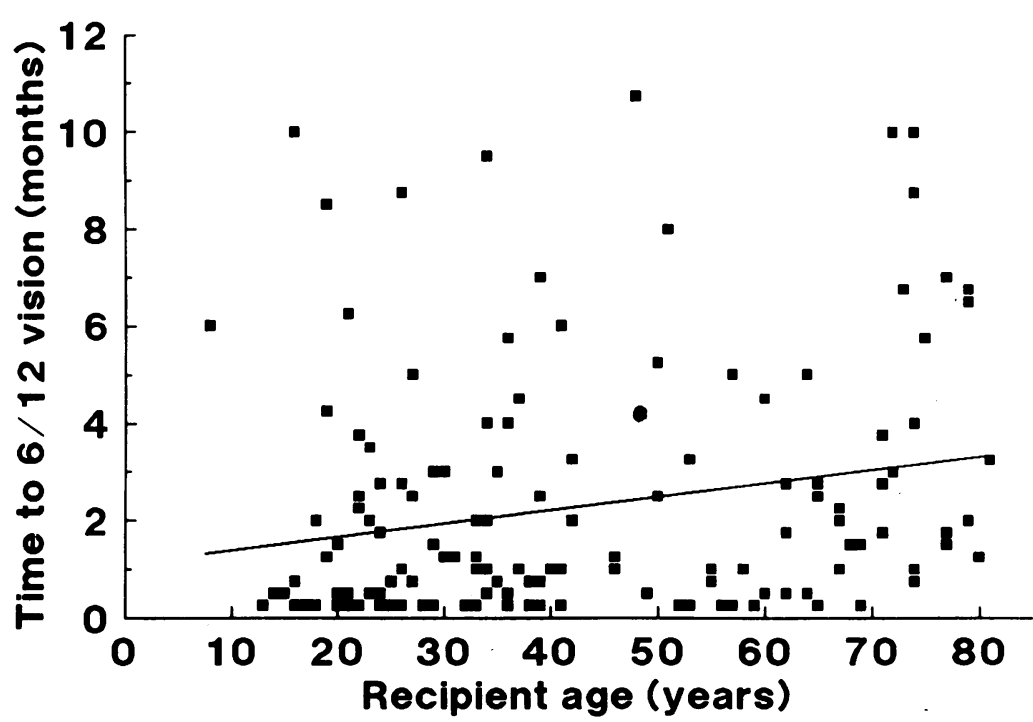

Figure 6: Scattergram of time to 6/12 vision versus age of recipient. There is significant correlation as shown by the linear regression line.

Individual grafts that are equally satisfactory from a clinical point of view may show wide variation in these measures. Laboratory based tests of corneal function can provide a basis by which various cornea storage techniques can be evaluated, , $^{5-8}$ but an ideal clinically applicable method of assessing endothelial cell viability has yet to be developed. ${ }^{12}$ In our patients further follow-up may proyide information on long term decompensation and may show, for example, an increased rate of late failure in grafts from older donors.

Patients in this study showed a very wide variation in the time that was taken to reach a postoperative acuity of $6 / 12$. No significant correlation was found with the known donor parameters, but there was a significant delay in recovery of vision with increasing recipient age. However, this accounted for only a very small part of the variation in time to $6 / 12$. Other factors, not identified here, such as reduced macular function in the elderly may be important. Improved techniques are needed to assess both donor corneas and also more of the variable host factors, such as inflammatory response and the presence and time course of cystoid macular oedema.

Although this study failed to find clinical advantage in using corneas from younger donors or from corneas stored for shorter periods in any one storage system, such corneas remain theoretically preferable. The use of McCareyKaufman and K-Sol storage media, with the associated increased time of storage, facilitated the scheduling of surgery, but were associated with an increased risk of primary graft failure.

We thank Darwin Minassian for his statistical analysis and the surgeons of Moorfields Eye Hospital for permission to report their cases.

1 Forster RK, Fine $M$. Relation of donor age to success in penetrating keratoplasty. Arch Ophthalmol 1971; 85: 42-7.

The Australian Graft Registry 1988 Report. Ed Williams KA Sawyer MA, White MA, et al. Published by Lions Save Sight Foundation, Flinders Medical Centre, Bedford Park 5042 Foundation, Flinde

3 Beijer-Dominicus JA, van Rij G, Beekhuis WH, et al. Visual acuity of patients transplanted with corneas transplanted in tissue culture and in McCarey-Kaufman medium. In Cavanagh HD, ed. The cornea: transactions of the World Congress on the Cornea III. New York: Raven Press, 1988: 115-6.

4 Kaufman HE, Varnell ED, Kaufman S, Beuerman RW, Barron BA. K-Sol preservation. Am $\mathcal{J}$ Ophthalmol 1985; 100: 299-304

5 Lass JH, Greiner JV, McBride M, Morgan DC, Medcalf SK, Glonek T. Effects of intermediate term storage on corneal metabolism: K-Sol versus CSM. In: Cavanagh HD, ed. The cornea: transactions of the World Congress on the Cornea III. cornea: transactions of the World Congres:

6 Lindstrom RL, Skelnik DL. Corneal preservation at $4^{\circ} \mathrm{C}$ with chondroitin sulphate containing medium. In: Cavanagh chondroitin sulphate containing medium. In: Cavanagh HD, ed. The cornea: transactions of the World Con

7 Busin M, Yau CW, Avni I, Kaufman HE. Comparison of $\mathrm{K}$-Sol and $\mathrm{M}-\mathrm{K}$ medium for cornea storage: results of penetrating keratoplasty in rabbits. $\mathrm{Br} \mathcal{F}$ Ophthalmol 1986 70: $860-3$.

8 Stein RM, Bourne WM, Campbell J. Chondroitin sulphate for corneal preservation at $4^{\circ} \mathrm{C}$ : evaluation by electron microscopy. Arch Ophthalmol 1986; 104: 1358-61.

9 Jaffe NS, Clayman HM, Jaffe MS. Cystoid macular edema after intracapsular and extracapsular extraction with and without an intraocular lens. Ophthalmology 1982; 89: 25-9.

10 Bourne WM. Endothelial cell survival on transplanted human corneas preserved at $4^{\circ} \mathrm{C}$ in $2.5 \%$ chondroitin sulphate for one to 13 days. Am $\mathcal{f}$ Ophthalmol 1986; 102: 382-6.

11 Halliday BL, Ross JE. Comparison of two interferometers for predicting visual acuity in patients with cataract. for predicting visual acuity in

12 Wilson SE, Bourne WM. Corneal preservation. Surv Ophthalmol 1989; 33: 237-59. 